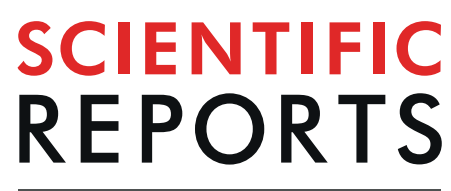

natureresearch

Check for updates

\title{
Amelioration of Physical Properties and Printability of Paper Coated with $\mathrm{N}$-methylated Chitosan
}

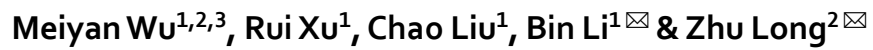

In offset printing process, poor mechanical properties and printability of paper substrate usually result in printing problems, low quality of print and waste of paper materials. Therefore, many researches focus on the quality improvement of paper substrates using suitable additives. In this work, N-methylated chitosan, including N, N-dimethyl chitosan (DMC) and N, N, N-trimethyl chitosan (TMC), were prepared and employed as coating agents to ameliorate the mechanical properties and printability of paper sheets. Analysis results showed that the mechanical strength of coated papers with DMC and TMC were largely improved, because the fibers with negative charges were prone to form electrostatic bonding with the positively charged $\mathrm{N}$-methylated chitosan, thus enhancing paper strength. Particularly, compared with chitosan and DMC, the TMC-coated paper exhibited better mechanical properties, printability and surface properties due to the high cationic charge density of TMC. Therefore, surface coating with TMC is of great benefit to decrease the printing problem of paper sheets and enhance the operation speed in offset printing. This work provides a valuable reference for the amelioration of the printability and physical properties of high-quality paper products for many promising applications.
\end{abstract}

Up to now, paper sheet made by lignocellulosic fibers is one of the most widely used materials in the world due to its good recyclability and biodegradability ${ }^{1}$. However, the mechanical strength and printability of paper still need to be improved to reduce the printing problem in offset, such as paper breaking, picking telegraphing, and poor color reduction ${ }^{2}$. To overcome these issues, one effective method is to coat polymer onto paper sheet. Starch $^{3,4}$, chitosan ${ }^{5,6}$, beeswax ${ }^{7}$, soy protein $^{8}$ and polyvinyl alcohol (PVA) ${ }^{9}$ have stimulated considerable interest as high-efficiency coating agents. Among them, chitosan with the structure of (1-4)-linked 2-acetamido-2deoxy-d-glucopyranose is the only positively charged natural biodegradable polymer, which is fully or partially deacetylated chitin ${ }^{10}$. The protonation of amino groups in chitosan under acidic conditions leads to the formation of positively charged surface of chitosan ${ }^{11}$. In papermaking process, the bonding between the fibers of paper can be increased by the electrostatic adsorption between the positive charge of amino groups on chitosan chain and the negative charge on the surface of fiber in water ${ }^{4,12,13}$. Therefore, chitosan can be used as a wet-end additive to increase the strength of paper. Moreover, chitosan with good film-forming and antibacterial properties is a suitable cationic polymer as a coating material ${ }^{14}$. The strength and the barrier properties against water vapor and oxygen of paper substrates can be largely increased after coated by chitosan ${ }^{6,15}$.

However, chitosan is insoluble in neutral and alkaline conditions due to the amino group in chitosan with a pKa value of 6.5 , which limits its application. $\mathrm{N}$-methylation is a way to directly modify chitosan by introducing alkyl groups onto $\mathrm{N}$ site of the structure of chitosan. It has been demonstrated that the hydrogen bonding of $\mathrm{N}-\mathrm{H}$ was reduced and the positive charge of chitosan in an acid solution was increased after $\mathrm{H}$ atoms on $\mathrm{N}$ substituted by methyl ${ }^{16,17}$. N, N-dimethyl chitosan (DMC) and N, N, N-trimethyl chitosan (TMC) are N-methylated chitosan by introducing two and three methyl groups onto $\mathrm{N}$ site of chitosan, respectively. The chemical modification of chitosan can reduce the rigid structure of chitosan, hence improving its solubility for end applications. According to the previous reports, there are three kinds of $\mathrm{N}$-methylation agents to produce TMC, including dimethyl sulfate, methyl iodide and dimethyl carbonate ${ }^{18-20}$. Methyl iodide is a carcinogenic and very expensive

${ }^{1}$ CAS Kay Laboratory of Biofuels, Dalian National Laboratory for Clean Energy, Qingdao Institute of Bioenergy and Bioprocess Technology, Chinese Academy of Sciences, Qingdao, 266101, China. ${ }^{2}$ Key Laboratory of Eco-textiles, Ministry of Education, Jiangnan University, Wuxi, 214122, China. ${ }^{3}$ Key Laboratory of Pulp and Paper Science \& Technology of Ministry of Education/Shandong Province, Qilu University of Technology (Shandong Academy of Sciences), Jinan, 250353, China. ${ }^{凶}$ e-mail: libin@qibebt.ac.cn; longzhu@jiangnan.edu.cn 


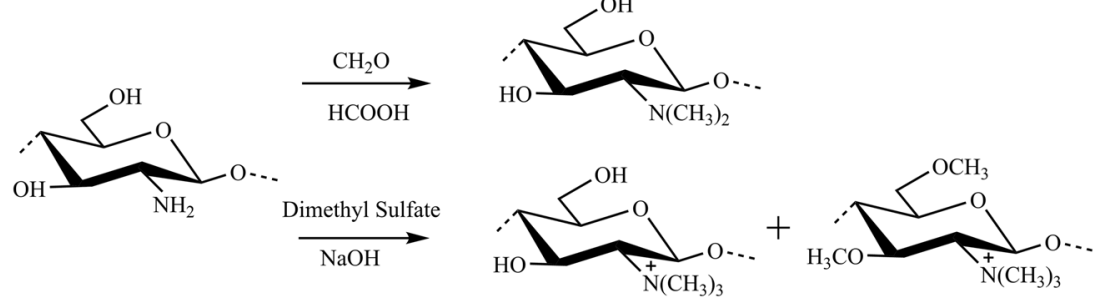

Figure 1. Synthesis routes of DMC (up) and TMC (down).

solvent, which is generally used in the high value-added filed ${ }^{21}$, such as pharmaceutical engineering, analytical chemistry, organic synthesis, etc. Moreover, although dimethyl carbonate was reported as a green solvent, the reaction of dimethyl carbonate usually requires high temperature and high pressure due to the low reactivity ${ }^{22}$. In our previous work, TMC was prepared using dimethyl carbonate ${ }^{20}$. The preparation process of TMC was firstly conducted to produce dimethyl chitosan (DMC), and then DMC in an ionic liquid was reacted with dimethyl carbonate under high temperature $\left(150^{\circ} \mathrm{C}\right)$. Compared with dimethyl carbonate, dimethyl sulfate can be used to directly produce TMC from chitosan in an alkaline solution without the assisting of ionic liquid, and the required temperature for the reaction is relatively lower (room temperature to $70^{\circ} \mathrm{C}$ ) ${ }^{18}$. Thus, this low-priced one step approach using dimethyl sulfate as a N-methylation agent was employed to prepare TMC in this work.

Nowadays, TMC, a soluble quaternary ammonium salt of chitosan, is mostly used as mucosal permeation enhancers $^{23,24}$, nonviral vectors ${ }^{25}$, and antimicrobial agent ${ }^{26}$. In addition, it has also been reported that TMC nanoparticles could be attached to viscose cellulose fiber as a potential drug delivery system ${ }^{27}$, and TMC was also applied onto cotton fabric by the pad-dry cure method for progressively improving the absorbency, dyeing behavior and wrinkle recovery ${ }^{28}$.

Therefore, it is expected that both DMC and TMC have great potential to be used as treatment agents for paper sheets, which are also formed by cellulose. In the present work, the effect of N-methylated chitosan on the mechanical properties, printability and surface property of the coated paper was comprehensively investigated. The results will be of great importance for the amelioration of printing efficiency and end paper quality, particularly for the high-quality packaging and decoration applications.

\section{Results and discussion}

Structure and water solubility analysis of methylated chitosan. DMC and TMC were prepared by the reaction of the methylation reaction, and the synthesis routes of DMC and TMC are shown in Fig. 1. Then, the structure of chitosan and N-methylated chitosan (DMC and TMC) was firstly investigated by FTIR spectroscopy. As shown in Fig. 2a, the broad peak around $3437 \mathrm{~cm}^{-1}$ belongs to the stretching vibrations of $\mathrm{O}-\mathrm{H}$ and N-H bonds, and the peaks at 2906 and $2884 \mathrm{~cm}^{-1}$ are attributed to the asymmetrical stretching C-H bonds ${ }^{29}$, which is observed in all spectra. Moreover, the peak at $1380 \mathrm{~cm}^{-1}$ is assigned to $\mathrm{C}-\mathrm{N}$ bonds in the structure of chitosan ${ }^{15}$. In addition, the two peaks at $1643 \mathrm{~cm}^{-1}$ and $1595 \mathrm{~cm}^{-1}$ in the spectrum of chitosan belong to amide I and amide II, respectively. Amide I mainly derives from the $\mathrm{C}=\mathrm{O}$ bonds vibrations of the amide and amide II is mainly dependent on the angular deformation of $\mathrm{N}-\mathrm{H}$ bending ${ }^{20}$. Compared to chitosan, there are two new peaks at $1536 \mathrm{~cm}^{-1}$ and $1460 \mathrm{~cm}^{-1}$ caused by $\mathrm{CH}_{2}$ and $\mathrm{CH}_{3}$ for DMC and TMC. Simultaneously, the peak at $1595 \mathrm{~cm}^{-1}$ assigning to N-H bonds disappears in the spectra of DMC and $\mathrm{TMC}^{30}$. Simultaneously, there is a new peak at $835 \mathrm{~cm}^{-1}$ in the spectra of methylated chitosan due to C-H out-of-plane deformation ${ }^{31}$. These phenomena indicate that methyl groups are introduced to the amino structure of chitosan after methylation reaction ${ }^{32,33}$.

${ }^{1} \mathrm{H}$ NMR spectra of chitosan and methylated chitosan were tested for further characterization, as shown in Fig. 2b. From the spectrum of chitosan, the signal between 4.75 and $5.25 \mathrm{ppm}$ is attributed to hydrogen bonded to the anomeric carbon $1^{34}$, the signals between 3.10 and $4.15 \mathrm{ppm}$ corresponds to hydrogen atoms of the glucopyranose ring, and the signal at $2.35 \mathrm{ppm}$ is assigned as hydrogen atoms of the acetyl group ${ }^{35}$. Compared to chitosan, there is a new signal at $3.0 \mathrm{ppm}$ in the spectrum of DMC, which belongs to hydrogen atoms of dimethyl group ${ }^{20}$. In the spectrum of TMC, the new signals can be observed at 2.9 and $3.1 \mathrm{ppm}$, which are attributed to the hydrogen atoms of methyl groups and dimethyl groups ${ }^{36}$, respectively. Furthermore, the TMC spectrum shows the signals at $3.25 \mathrm{ppm}$ and $3.45 \mathrm{ppm}$, which are assigned to hydrogen atoms of trimethyl groups and methoxy group $\left(-\mathrm{OCH}_{3}\right)$, bonded to carbon 3 and carbon $6^{18}$, respectively. This result is due to the weak selectivity of dimethyl sulfate to $\mathrm{NH}_{2}$ and $\mathrm{OH}$ groups of chitosan in the process of methylation ${ }^{37}$. Therefore, the structure of DMC and TMC were proved by ${ }^{1} \mathrm{H}$ NMR and FTIR results.

In addition, the degree of substitution (DS) of DMC and the degree of quaternization (DQ) of TMC can be calculated by the integral area of the hydrogen atoms from the ${ }^{1} \mathrm{H}$ NMR spectrum according to formula (1) and formula (2), respectively. Meanwhile, the degree of O-methylation (DO) of TMC can also be calculated with the same method. According to the calculation of formulas (1) and (2), the DS of DMC was $91.0 \%$, while the DQ and DO of TMC were $5.56 \%$ and $13.5 \%$, respectively.

$$
\mathrm{DS}=\frac{\frac{I_{D M C}}{6}}{I_{H 1}} \times 100 \%
$$



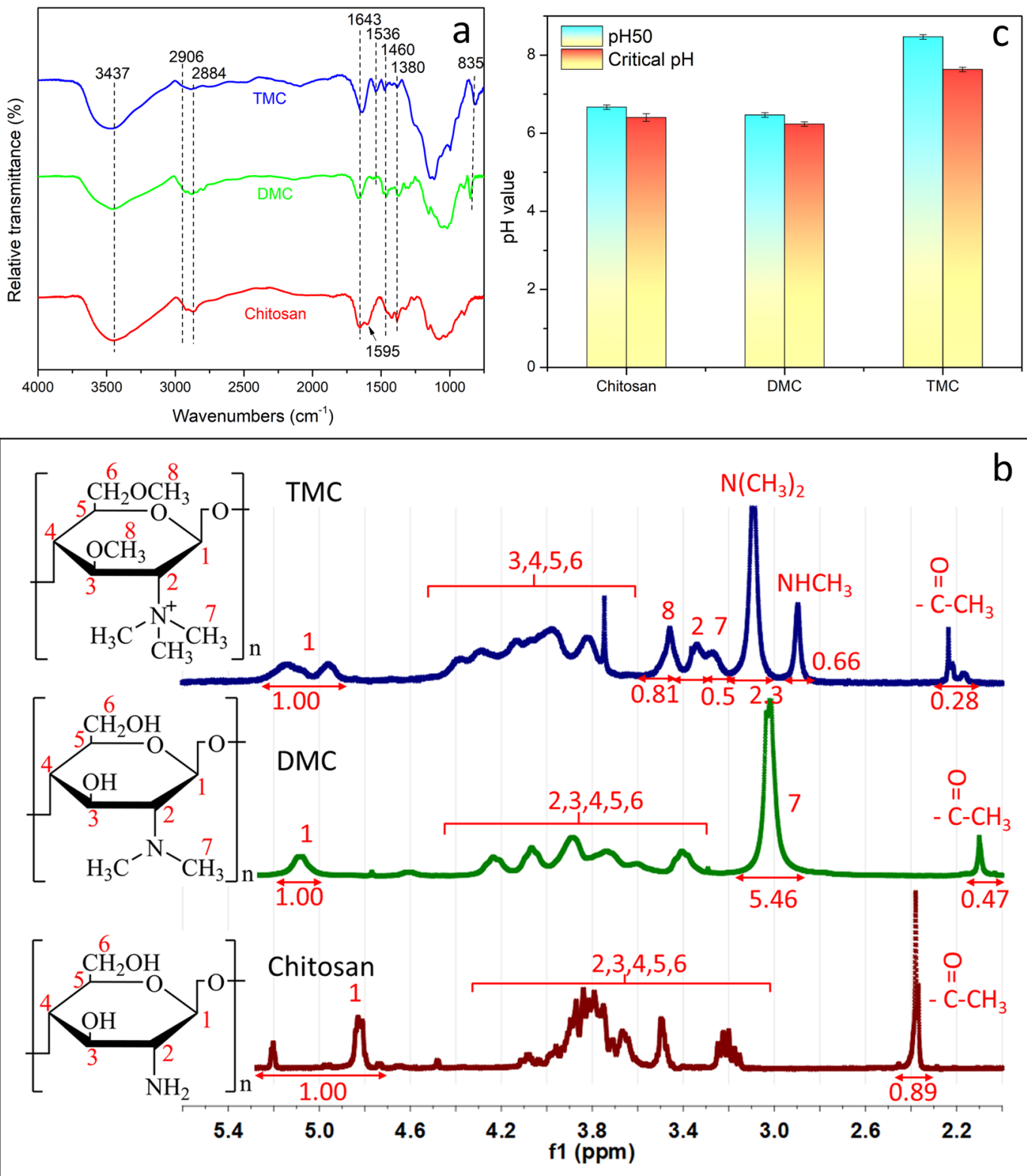

Figure 2. Structure and water solubility analysis of N-methylated chitosan. (a) FTIR spectra of chitosan and $\mathrm{N}$-methylated chitosan. (b) ${ }^{1} \mathrm{H}$ NMR spectra of chitosan and N-methylated chitosan. (c) Solubility of chitosan and N-methylated chitosan.

Where $I_{D M C}$ is the integral of the hydrogen atoms on dimethyl group, and $I_{H 1}$ is the integral of the hydrogen atoms bonded to Carbon $1^{20}$.

$$
\mathrm{DQ}=\frac{\frac{I_{T M C}}{9}}{I_{H 1}} \times 100 \%
$$

Where $I_{T M C}$ is the integral of the hydrogen atoms on the quaternary ammonium group.

The water solubility results of $\mathrm{N}$-methylated chitosan are shown in Fig. 2c. The $50 \%$ of transmission rate of chitosan solution ( $\mathrm{pH} 50$ ) is at the $\mathrm{pH}$ of 6.7 , while the $98 \%$ of transmittance rate of chitosan solution (Critical $\mathrm{pH}$ ) is at the $\mathrm{pH}$ of 6.4. Compared with chitosan, the solubility of DMC has no obvious change, but the pH50 and Critical $\mathrm{pH}$ of TMC are 8.5 and 7.6, respectively. This result suggests that the solubility of chitosan derivative with quaternary ammonium can be greatly improved ${ }^{38}$, and it can be dissolved in the $\mathrm{pH}$ range from 1 to 7.6 at the concentration of $2 \mathrm{mg} / \mathrm{mL}$, which effectively expand the scope of solubility. Good water solubility of TMC under neutral conditions is of great help to reduce the equipment corrosion and the loss of paper strength by acid solution, thus reducing the production cost of high-quality paper sheets. 

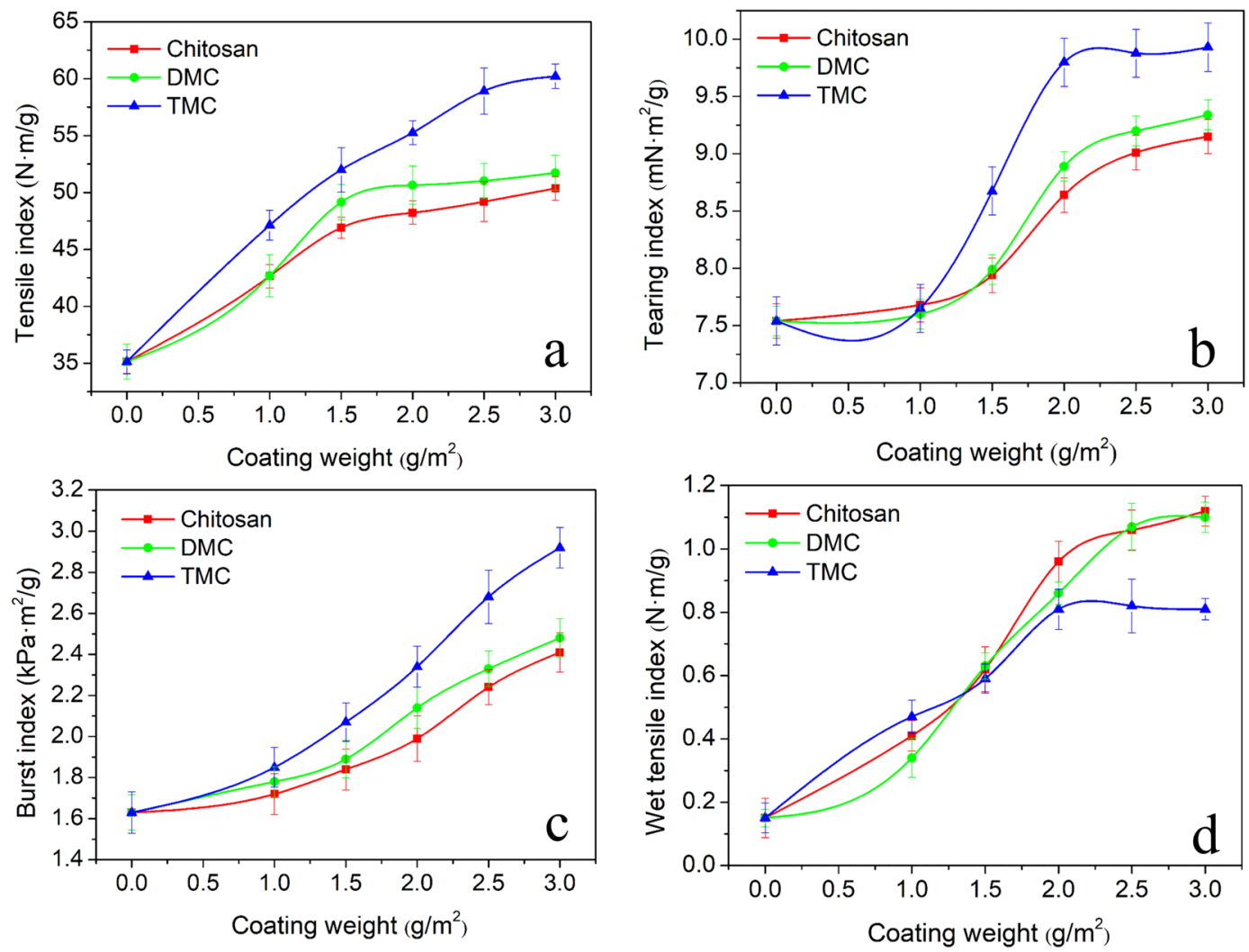

Figure 3. Mechanical properties of coated papers. (a) Tensile index. (b) Tearing index. (c) Burst index. (d) Wet tensile index.

Mechanical properties of coated papers. As known, the mechanical properties of paper sheets are of great importance for the manufacture and printing process ${ }^{39,40}$. Therefore, the mechanical properties of coated papers, including tensile index, burst index, tearing index and wet tensile index, were tested to evaluate the effect of coating with $\mathrm{N}$-methylated chitosan. As reported, the mechanical properties of paper sheets depend on the quality of fiber itself and the bonding strength between fibers ${ }^{41,42}$. The bonding strength between fibers can be increased by the supplement of additives in pulp ${ }^{43}$ or surface coating on paper sheets ${ }^{9}$.

As shown in Fig. 3a, the tensile index of paper sheets is largely increased with the increase of coating weight. Especially, the tensile index of TMC-coated paper increased from 35.1 to $60.2 \mathrm{~N} \cdot \mathrm{m} / \mathrm{g}$ with the coating weight of $3.0 \mathrm{~g} / \mathrm{m}^{2}$, which is increased by $71.5 \%$. Meanwhile, the trend of the tearing index of paper sheet coated with methylated chitosan is similar with the tensile index of coated paper, and the tearing index of chitosan, DMC and TMC coated paper with the coating weight of $3.0 \mathrm{~g} / \mathrm{m}^{2}$ is increased by $21.4 \%, 23.9 \%, 31.7 \%$, respectively (Fig. 3b). Moreover, Fig. $3 \mathrm{c}$ shows that the growth rate of the burst index is slow with a lower coating weight of methylated chitosan $\left(<1.5 \mathrm{~g} / \mathrm{m}^{2}\right)$, but there is a rapid increase of the burst index with the coating weight higher than $1.5 \mathrm{~g} /$ $\mathrm{m}^{2}$. When the coating weight is the maximum dose of $3.0 \mathrm{~g} / \mathrm{m}^{2}$, the burst index of TMC-coated paper also arrives at maximum, which is increased by $79.1 \%$ compared with the control. In addition, when the coated weight is $3.0 \mathrm{~g} / \mathrm{m}^{2}$, the wet strength of paper sheets coated with chitosan, DMC and TMC increased 6.5, 6.3 and 4.4 times, respectively (Fig. 3d).

In addition, the tensile index of coated paper with TMC (this work) is compared with that of coated papers with other typical regents in Fig. 4. As can be seen, the tensile index of the coated paper with TMC is higher than that of the coated papers with starch ${ }^{44}$, oxidized $\operatorname{starch}^{44}$, chitosan/caseinate ${ }^{6}$, chitosan $/ \mathrm{TiO}_{2}{ }^{45}, \operatorname{triclosan} / \mathrm{CNF}$ (cellulose nanofibrils) ${ }^{46}$ or HPMC (hydroxypropyl methylcellulose) ${ }^{47}$, and lower than that of the coated papers with $\mathrm{CNF} /$ nanoclay $^{48}$, and guanidine-grafted CMC (carboxymethyl cellulose ${ }^{49}$. However, although the used paper substrate had a lower tensile index, the increasing rate $(71.5 \%)$ of tensile index for the TMC-coated paper (with coating weight of $3.0 \mathrm{~g} / \mathrm{m}^{2}$ ) was clearly higher than the $\mathrm{CNF} /$ nanoclay $\left(14.3 \%\right.$, coating weight $10.0 \mathrm{~g} / \mathrm{m}^{2}$ ) and guanidine-grafted CMC coated papers $\left(16.7 \%\right.$, coating weight $\left.2.1 \mathrm{~g} / \mathrm{m}^{2}\right)$. Therefore, TMC is superior to some commonly used coating agents, such as starch, HPMC, for improving the mechanical strength of the coated paper.

To investigate the mechanism of the improvement of paper strength, the ionic charge density of different additives was tested accordingly. The ionic charge density of additives with the concentration of $0.1 \mathrm{wt} . \%$ in acid solution are illustrated in Table 1 . It was measured under $\mathrm{pH}$ value of 3,5 and 7 in consideration of acid soluble chitosan. As expected, the cationic charge density of TMC solution is higher than that of chitosan and DMC solutions under acid and neutral conditions. Especially, with $\mathrm{pH}$ value of 3 and 5 , the cationic charge density of TMC solution is increased by $34.6 \%$ and $33.8 \%$, respectively, in comparison with chitosan. The fibers with the 


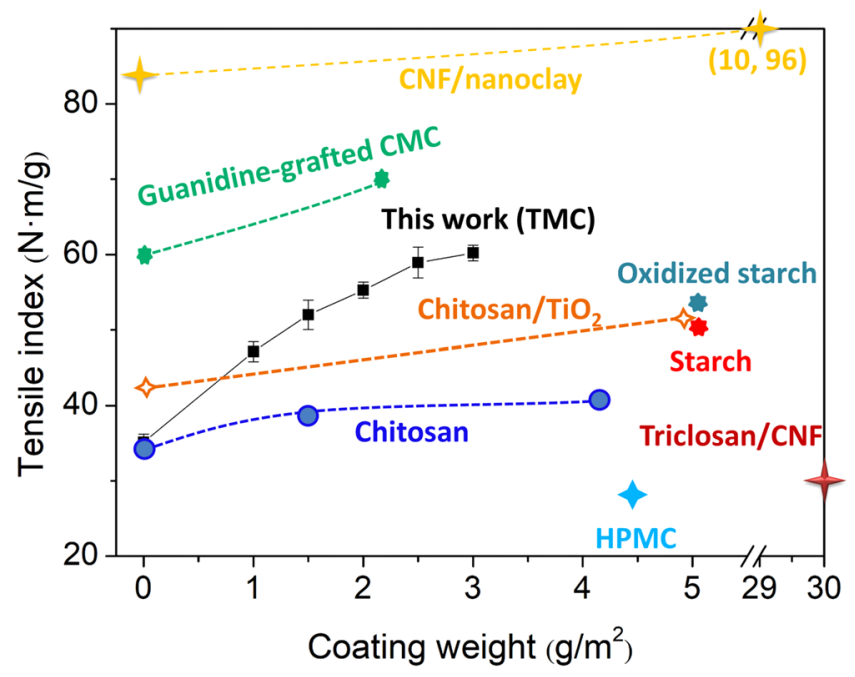

Figure 4. Comparison of the tensile index of the coated papers with different coating reagents. Note: Chitosan ${ }^{63}$, Starch and oxidized starch ${ }^{44}$, Chitosan/caseinate ${ }^{6}$, Chitosan/TiO ${ }_{2}^{45}$, Triclosan/CNF (Cellulose nanofibrils ${ }^{46}$, HPMC (Hydroxypropyl methylcellulose) ${ }^{47}, \mathrm{CNF} /$ nanoclay $^{48}$, guanidine-grafted CMC (Carboxymethyl cellulose $)^{49}$.

\begin{tabular}{|l|l|l|l|}
\hline \multirow{2}{*}{$\mathbf{p H}$ value } & \multicolumn{4}{|l|}{ Cationic charge density $(\mathbf{m e q} / \mathbf{g})$} \\
\cline { 2 - 4 } & Chitosan & DMC & TMC \\
\hline $\mathrm{pH}=3$ & $0.205 \pm 0.002$ & $0.234 \pm 0.002$ & $0.276 \pm 0.001$ \\
\hline $\mathrm{pH}=5$ & $0.201 \pm 0.001$ & $0.228 \pm 0.003$ & $0.269 \pm 0.002$ \\
\hline $\mathrm{pH}=7$ & Not detected & $0.053 \pm 0.004$ & $0.120 \pm 0.002$ \\
\hline
\end{tabular}

Table 1. Cationic charge density of chitosan and N-methylated chitosan.

\begin{tabular}{|l|l|l|}
\hline Paper sample & $\begin{array}{l}\text { Printing speed } \\
(\mathbf{m} / \mathbf{s})\end{array}$ & $\begin{array}{l}\text { Surface strength } \\
(\mathbf{m} / \mathbf{s})\end{array}$ \\
\hline Paper substrate & 5 & $2.79 \pm 0.03$ \\
\hline Chitosan-coated paper & 5 & $3.00 \pm 0.02$ \\
\hline DMC-coated paper & 5 & $3.54 \pm 0.03$ \\
\hline TMC-coated paper & 5.5 & $3.44 \pm 0.03$ \\
\hline
\end{tabular}

Table 2. Surface strength of coated papers.

negative charges are prone to form electrostatic bonding with the cationic charged N-methylated chitosan with the increase of introducing methyl groups onto amino group of chitosan ${ }^{50}$. This effect leads to the enhancement of the adhesion strength of the fibers ${ }^{51}$. Therefore, TMC is a suitable and effective dry strengthening agent to improve the mechanical properties of paper sheet. Yet, chitosan coating yields a better wet tensile strength of coated paper in comparison with methylated chitosan (Fig. 3d), which is probably due to the insolubility of chitosan in water ${ }^{15}$.

Printability of coated paper. Printability of paper sheet plays a decisive role in the quality of print. After coating on the surface of paper sheets, the coating properties of coated paper, such as the surface strength, ink absorbency and liquid absorbency, are the crucial factors affecting printability of paper sheet ${ }^{52}$. Therefore, it is important to investigate the effect of those factors on paper printability after coating with chitosan and $\mathrm{N}$-methylated chitosan.

Surface strength of paper sheets refers to the bonding between filler or fiber fines and fiber materials on the surface of paper substrate ${ }^{53}$. Low surface strength of paper sheets can result in easy absorption between filler or fiber fines and ink, and then the picking and linting on the surface of paper sheets can be observed due to the tension of ink in printing process ${ }^{54}$. Surface strength of coated paper are exhibited in Table 2 . After coated with chitosan and $\mathrm{N}$-methylated chitosan, the surface strength of all paper samples was improved. In particular, when printing speed maintains $5.5 \mathrm{~m} / \mathrm{s}$, the surface strength of TMC-coated paper $(3.44 \mathrm{~m} / \mathrm{s})$ is still higher than that $(3 \mathrm{~m} / \mathrm{s})$ of chitosan-coated paper. These results suggest that effective adsorption happens between TMC and the surface of paper sheet, and TMC plays a bridge role between fibers to improve the degree of the combination of fibers ${ }^{24}$. Fiber fines and filler can firmly stay on the surface of paper sheets even at a high printing speed ${ }^{55}$. Thus, 


\begin{tabular}{|l|l|l|l|}
\hline Paper sample & $\mathbf{Y}_{\mathbf{0}}(\mathbf{\%})$ & $\mathbf{Y}_{\mathbf{1}} \mathbf{( \% )}$ & $\mathbf{Y}(\mathbf{\%})$ \\
\hline Paper substrate & $75.65 \pm 0.01$ & $41.67 \pm 0.03$ & 44.92 \\
\hline Chitosan-coated paper & $73.15 \pm 0.05$ & $42.11 \pm 0.06$ & 42.43 \\
\hline DMC-coated paper & $74.26 \pm 0.03$ & $34.96 \pm 0.04$ & 52.92 \\
\hline TMC-coated paper & $76.90 \pm 0.05$ & $33.44 \pm 0.05$ & 56.51 \\
\hline
\end{tabular}

Table 3. Ink absorption ability of coated papers.
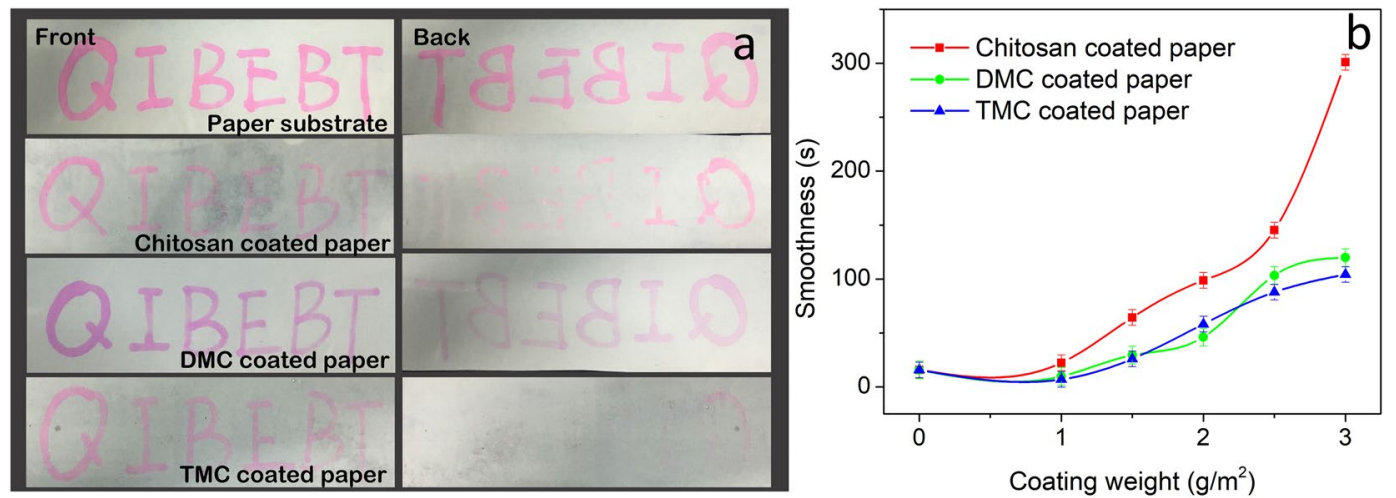

Figure 5. (a) Liquid absorbency of coated papers with chitosan and N-methylation chitosan. (b) Effect of coating weight on smoothness of coated paper.

due to the increasing of the surface strength of papers by coating with TMC, the operation speed of printing press can be increased, which is of great importance for the improvement of production efficiency.

Ink absorbency of coating is an important factor in printing. If the ink absorption of coating is too high, it can cause the gradual loss of color printing, the poor color reducibility, telegraphing and other issues. If it is too low, it can cause the printing problems of dot gain and smearing on the back ${ }^{52}$. In this work, the reflex factor of green ray before and after ink absorption was tested (Table 3), and $\mathrm{K} \& \mathrm{~N}$ ink absorption value of coated papers with chitosan and $\mathrm{N}$-methylated chitosan was calculated by the following formula (3).

$$
Y=\frac{Y_{0}-Y_{1}}{Y_{0}} \times 100 \%
$$

Where $Y$ is the K\&N ink absorption value, $Y_{0}$ is the reflex factor of green ray and $Y_{1}$ is the reflex factor of green ray after ink absorption.

It can be observed that when the coating weight is $1.5 \mathrm{~g} / \mathrm{m}^{2}$, the ink absorption value of chitosan-coated paper is lower than that of paper substrate due to the good oil-resistance of chitosan ${ }^{56}$. However, compared with chitosan-coated paper, the coated papers with N-methylated chitosan exhibit applicable ink absorbency. This phenomenon is because N-methylated chitosan (DMC and TMC) coated papers have suitable smoothness for absorption ink in printing process. In addition, because a large amount of positive charges is equipped on the surface of TMC (Table 1), the ink binder made of the negatively charged resin or oil can be absorbed by positively charged TMC. Thus, ink can be firmly absorbed on the surface of TMC-coated paper. Therefore, TMC-coated papers have better ink absorbency for offset printing in comparison with chitosan and DMC.

In offset printing process, fountain solution should be first adsorbed on the paper, and then ink can be printed according to the principle of oil-water incompatibility ${ }^{57}$. As a result, paper sheet needs a certain liquid absorption to accelerate the permeation of fountain solution. However, excessive liquid absorption is easy to cause the printing trouble of telegraphing ${ }^{58}$. After coating with chitosan and $\mathrm{N}$-methylated chitosan, the new forming film on the surface increases the barrier properties of paper and simultaneously decreases the liquid permeation. Liquid permeation and coating uniformity of coated paper can be measured according to the liquid permeation test. The front and back of coated papers after brushing with rhodamine B solution are photographed in Fig. 5a. Compared with paper substrate, the pale brushing imprint and less solution permeation of chitosan-coated paper can be seen. While DMC-coated paper not only exhibits clear brushing imprint and high color reduction in the front, but also shows low degree of permeation in the back. Particularly, the permeation degree of TMC-coated paper is even lower than that of DMC-coated paper. These results indicate that TMC-coated paper has a suitable liquid absorbency, and the printing problem of telegraphing is less likely to happen in the event of TMC as a coating agent.

Surface properties of coated paper. Smoothness of paper refers to the leaking time of a certain volume of air going through between the smooth glass plate and the paper sheet under a certain vacuum degree. For those paper sheets which need to be printed, the printing density and the color reproducibility of coated papers are related to the smoothness of paper sheets. Smoothness of coated papers largely depends on the degree of coating 

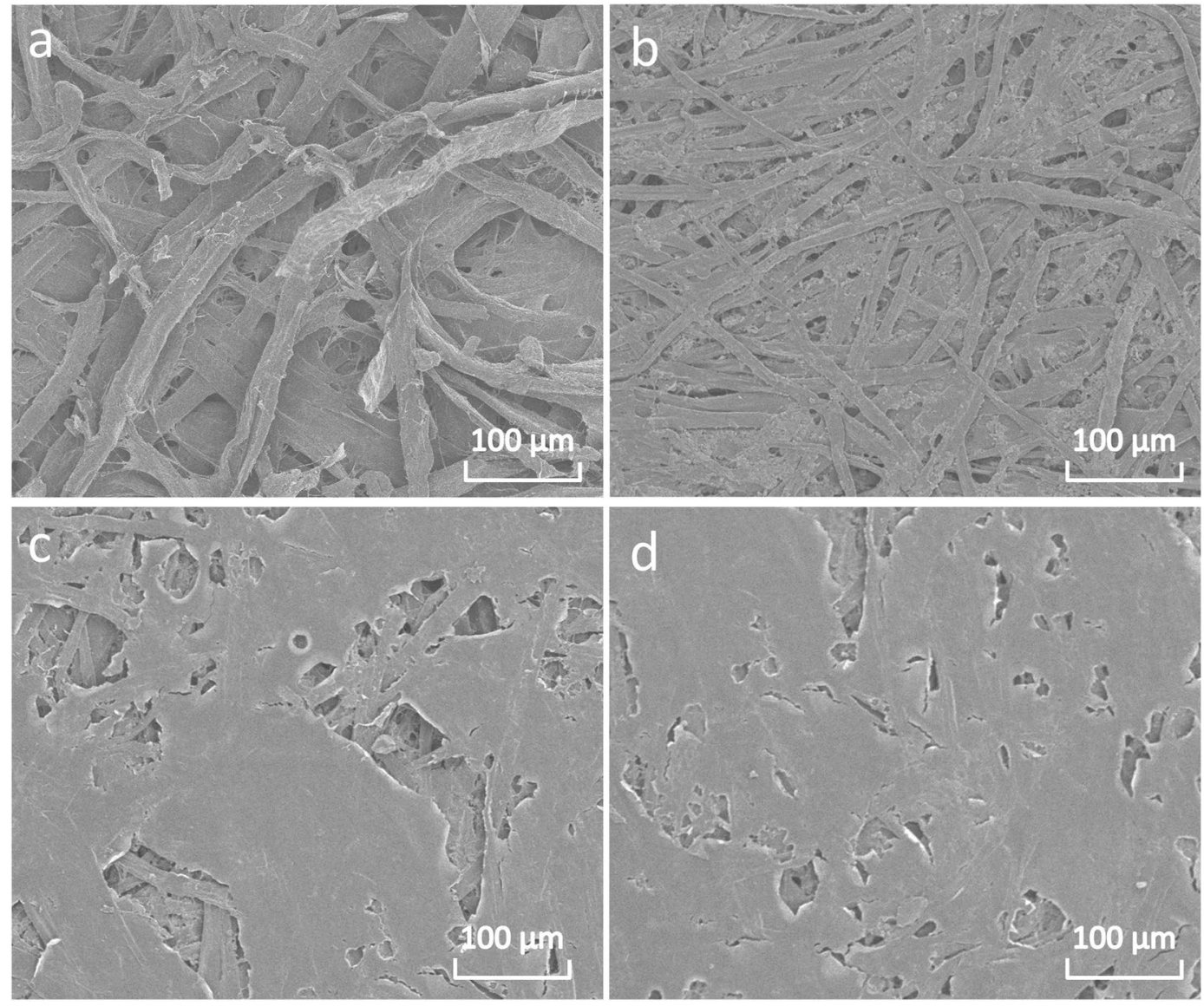

Figure 6. SEM images of coated papers. (a) Paper substrate. (b) Chitosan-coated paper. (c) DMC-coated paper. (d) TMC-coated paper.

uniformity ${ }^{59}$. The smoothness of coated paper with chitosan and N-methylated chitosan are given in Fig. 5b. When the coating weight is less than $1.0 \mathrm{~g} / \mathrm{m}^{2}$, there is no obvious change in smoothness of coated paper with chitosan and $\mathrm{N}$-methylated chitosan. However, when the coating weight is larger than $1.0 \mathrm{~g} / \mathrm{m}^{2}$, the smoothness of coating is increased quickly, particularly for chitosan-coated paper. The smoothness of chitosan, DMC and TMC coated paper with the coating weight of $3.0 \mathrm{~g} / \mathrm{m}^{2}$ is increased 18.2, 6.7 and 5.7 times, respectively, compared to the control (paper substrate). This phenomenon is because chitosan, DMC and TMC solutions with different viscosity fail to evenly spread out over the surface of papers when the coating weight is lower, and then air can seep from cracks on the surface of paper sheets. However, when the coating weight is higher than $1.0 \mathrm{~g} / \mathrm{m}^{2}$, the surface of paper sheets can be wet by chitosan and $\mathrm{N}$-methylated chitosan and the formed coating is relatively uniform to stop the air flow, which is also can be seen in Fig. 6. Therefore, the smoothness of coated papers is largely increased by coating with TMC.

Scanning electron microscopy (SEM) was employed to study the surface of coated papers with the coating weight of $1.5 \mathrm{~g} / \mathrm{m}^{2}$. Fig. 6 a shows that the surface of fibers is smooth and large number of big holes between fibers on paper substrate. Fig. $6 \mathrm{~b}$ displays that the surface of chitosan-coated paper is only partly covered and the voids between fibers are decreased in comparison with paper substrate. However, there is more uniform coating on the surface of DMC and TMC-coated papers, and most of the fibers are covered by the coating (Fig. 6c,d). This phenomena are because chitosan solution is hard to form a uniform coating due to its high viscosity, while for DMC and TMC, more hydrogen atoms on amino groups of chitosan were substituted with methyl groups, leading to a better solubility and lower viscosity of $\mathrm{N}$-methylated chitosan ${ }^{38}$. Thus, both DMC and TMC solutions are easier to get a flat and uniform coating, leading to a better warp-resistance and increased strength of paper sheet after coating. In addition, the increased smoothness and more compact surface of paper are beneficial to improve the barrier properties of end products, which is good for the final application in high quality packaging for foods, medicine, or cosmetics, to replace plastics ${ }^{15}$.

\section{Conclusions}

In this work, N-methylated chitosan (i.e. N, N-dimethyl chitosan (DMC) and N, N, N-trimethyl chitosan (TMC)), were successfully prepared via methylation reactions. The chemical structure of DMC and TMC was determined by FTIR and ${ }^{1} \mathrm{H}$ NMR, and the degree of substitution of DMC and the degree of quaternization of TMC were calculated to be $91.0 \%$ and $5.6 \%$, respectively. Also, in order to improve the physical properties and printability of printing paper, chitosan, DMC, and TMC were used as coating agents to coat paper. Results showed 
that compared with chitosan and DMC, TMC as a coating agent could significantly improve the mechanical properties of paper sheets due to the higher cationic charge density and better solubility. When the coating weight was $3.0 \mathrm{~g} / \mathrm{m}^{2}$ (to dry paper), the tensile index, tearing index and burst index of TMC-coated paper increased by $71.5 \%$, $31.7 \%$ and $79.1 \%$, respectively, and this increasing rate of tensile index with TMC was clearly higher compared to the typical coating agents (such as chitosan, starch, cellulose nanofibrils) with the comparable coating weight reported in literature. Especially, the wet tensile index of TMC-coated paper increased 4.4 times compared to the control. Moreover, the surface strength, ink absorbency and liquid absorbency of TMC-coated paper were more suitable for offset printing at a higher printing speed. This was because TMC with good solubility could form a more uniform coating on the surface of paper sheet and then increase the bonding strength between fibers via electrostatic interaction. Therefore, TMC as a high-efficient coating agent can be used in the offset process to improve the quality and production efficiency of the printings.

\section{Materials and Methods}

Chitosan (made from $\alpha$-Chitin, $210 \mathrm{kDa}$, degree of deacetylation of $84.6 \%$ ), formaldehyde solution (37 wt.\%), formic acid (98 wt.\%), NaOH, and acetone were purchased from Sinopharm Chemical Reagent Co., Ltd., China. Dimethyl sulfate was purchased from Linyi Yuanbo Chemical Industry Co., Ltd. Bleached hardwood kraft pulp was a gift from Mudanjiang Hengfeng Paper Co., Ltd., China, and the main components of the pulp were $86.1 \%$ of cellulose and $13.9 \%$ of hemicelluloses. All materials and chemicals were used without any further modification.

DMC was prepared by the reaction of formaldehyde and chitosan under acidic conditions to form Schiff base via Eschweiler-Clarke reaction ${ }^{60}$. Briefly, $10 \mathrm{~g}$ chitosan, $30 \mathrm{~g}$ formic acid, $40 \mathrm{~g}$ formaldehyde solution, and $180 \mathrm{~g}$ deionized water (DI) were added in a three-neck round-bottom flask equipped with a reflux condenser. The solution was heated to $70^{\circ} \mathrm{C}$ and stirred magnetically for $118 \mathrm{~h}$. After reduced pressure distillation, $1 \mathrm{~mol} / \mathrm{L} \mathrm{NaOH}$ solution was employed to adjust the $\mathrm{pH}$ value to 12 and then a gel was obtained. Subsequently, the gel was washed with DI, and then re-dissolved in acetic acid solution with $\mathrm{pH}$ of 4 . The solution was dialyzed in DI for one week using regenerated cellulose membrane with a molecular weight cutoff of 8,000-12,000 Da. Finally, DMC was obtained after freeze drying.

The preparation method of TMC using dimethyl sulfate was previously reported by $\mathrm{Wu}^{20}$. Ten grams of chitosan was slowly added in $40 \mathrm{~mL}$ DI and $160 \mathrm{~mL}$ dimethyl sulfate with magnetic stirring and reflux condensing system. Then, $60 \mathrm{~g}$ of $\mathrm{NaOH}$ and $44 \mathrm{~g}$ of $\mathrm{NaCl}$ were added in the flask. The solution was heated to $70^{\circ} \mathrm{C}$ and a dramatic reaction was observed. After $10 \mathrm{~h}$ reaction, the $\mathrm{pH}$ value of solution was adjusted to 7 , and then dialyzed in DI for one week. Finally, the product of TMC was washed with acetone and then vacuum dried at $50^{\circ} \mathrm{C}$ for $1 \mathrm{~h}$.

The obtained samples of chitosan and methylated chitosan were prepared as $\mathrm{KBr}$ pellets and scanned in the range of $750-4000 \mathrm{~cm}^{-1}$ using a Fourier transform infrared spectrometer (FTIR, Nicolet iS10, USA). The samples of chitosan and methylated chitosan were dissolved in a solution consisting of $10 \% \mathrm{D}_{2} \mathrm{O}$ and $90 \% \mathrm{DCl}$ with the concentration of $10 \mathrm{~g} / \mathrm{L}$ in a water bath at $80^{\circ} \mathrm{C}$, and ${ }^{1} \mathrm{H}$ spectra were recorded on a Nuclear magnetic resonance spectrometer (NMR, Bruker AVANCE III $400 \mathrm{MHz}$, USA).

Chitosan and its methylated derivatives were dissolved in $2 \%$ acetic acid solution with the concentration of $2 \mathrm{mg} / \mathrm{mL}$. The $\mathrm{pH}$ value of the solution was adjusted by dipping $1 \mathrm{~N} \mathrm{NaOH}$ solution, and then the transmittance of the solution at $600 \mathrm{~nm}$ was recorded using a UV-vis spectrometer (Hitachi U-4100, Japan). Critical pH value was named as the $\mathrm{pH}$ when the transmission rate of the solution is more than $98 \%$, while $\mathrm{pH} 50$ was defined as the $\mathrm{pH}$ when the transmittance reaches $50 \%{ }^{61}$. The tests were conducted at least three times. In addition, the ionic charge density of additives was tested by the method of colloidal titration. This method is based on the stoichiometric combination of positive and negative colloid ions, and the potential change of solution can be measured by a Particle Charge Detector (Mütek BTG PCD-04 Travel, Germany).

Paper sheets with a basis weight of $60 \pm 2.5 \mathrm{~g} / \mathrm{m}^{2}$ were firstly prepared based on ISO 5269-1-79 using a paper sheet forming machine (Xianyang Taist Test Equipment Co., Ltd., ZQJ1-B-II, China). Chitosan and methylated chitosan were dissolved in acetic acid solution $(\mathrm{pH}=5.5)$ with the concentration of $2 \mathrm{wt} . \%$. Then, paper sheets were coated with chitosan and methylated chitosan solution using a wire bar coater (Yeke CCI-1000, China) with various coating weight from 0 to $3.0 \mathrm{~g} / \mathrm{m}^{2}$. The coated papers were dried at $70^{\circ} \mathrm{C}$ for $180 \mathrm{~s}$ and conditioned at $23 \pm 1{ }^{\circ} \mathrm{C}$ and $50 \pm 2 \%$ relative humidity for $12 \mathrm{~h}$ before tests. The tensile index, burst index, tearing index and wet tensile index of the coated papers were measured according to TAPPI standard procedures of T $404 \mathrm{wd}-03$, T 403 om-02, T 414 om-98, and T 456 om-03, respectively. The smoothness of coated paper with the coating weight of $1.5 \mathrm{~g} / \mathrm{m}^{2}$ was recorded according to T $479 \mathrm{~cm}-99$ (Bekk Method) using a Smoothness tester (TMI 58-05, USA). Each sample was measured five times for calculating the average value and standard deviations. The surface morphologies of coated papers with the coating weight of $1.5 \mathrm{~g} / \mathrm{m}^{2}$ were observed using a Scanning Electron Microscope (SEM, Hitachi S-4800, Japan). Before observation, gold spraying was treated on paper sheet samples under vacuum.

The surface strength of coated paper was measured using an IGT printability tester (C1-5, Netherlands) for the simulation of offset printing according to T $499 \mathrm{wd}-85$. The coating weight of offset papers was $1.5 \mathrm{~g} / \mathrm{m}^{2}$. The $\mathrm{K} \& \mathrm{~N}$ ink was absorbed by samples for $2 \mathrm{~min}$, and then wiped by cotton ball, the reflex factor of green ray of samples was recorded by a reflectance spectrophotometer (X-Rite 528, USA).

Liquid absorbency was also investigated with $0.5 \mathrm{wt}$.\% rhodamine B solution ${ }^{62}$. Rhodamine B was dissolved in anhydrous ethanol and then sealed for storage before use. The solution was coated on the coated paper (size $50 \mathrm{~mm} \times 100 \mathrm{~mm}$ ) using a cotton brush, and then after10 min, the permeation on the front and back of samples were photographed by a digital camera.

\section{Data availability}

The data used to support the findings of this study are available from the corresponding author upon request. 
Received: 23 February 2020; Accepted: 26 May 2020;

Published online: 18 June 2020

\section{References}

1. Mahadeva, S. K., Walus, K. \& Stoeber, B. Paper as a platform for sensing applications and other devices: A review. ACS Appl. Mater. Inter 7, 8345-8362 (2015).

2. Lestiani, R., Batchelor, W. \& Banham, P. Effect of paper and printing press variables on the rates of adhesion failure in the linting of offset printing. J. Adhes. Sci. Technol. 28, 1935-1948 (2014).

3. Ghasemian, A., Ghaffari, M. \& Ashori, A. Strength-enhancing effect of cationic starch on mixed recycled and virgin pulps. Carbohydr. Polym 87, 1269-1274 (2012).

4. Ashori, A., Cordeiro, N., Faria, M. \& Hamzeh, Y. Effect of chitosan and cationic starch on the surface chemistry properties of bagasse paper. Int. J Biol. Macromol. 58, 343-348 (2013).

5. Zhang, H., Shi, L. \& Feng, X. Use of chitosan to reinforce transparent conductive cellulose nanopaper. J Mater. Chem. C 6, 242-248 (2018).

6. Khwaldia, K., Basta, A. H., Aloui, H. \& El-Saied, H. Chitosan-caseinate bilayer coatings for paper packaging materials. Carbohydr. Polym 99, 508-516 (2014).

7. Zhang, W., Xiao, H. \& Qian, L. Beeswax-chitosan emulsion coated paper with enhanced water vapor barrier efficiency. Appl. Surf. Sci. 300, 80-85 (2014).

8. Arboleda, J. C., Niemi, N., Kumpunen, J., Lucia, L. A. \& Rojas, O. J. Soy protein-based polyelectrolyte complexes as biobased wood fiber dry strength agents. ACS Sustain. Chem. Eng. 2, 2267-2274 (2014).

9. Hamzeh, Y., Sabbaghi, S., Ashori, A., Abdulkhani, A. \& Soltani, F. Improving wet and dry strength properties of recycled old corrugated carton (OCC) pulp using various polymers. Carbohydr. Polym 94, 577-583 (2013).

10. Wang, H., Qian, J. \& Ding, F. Emerging chitosan-based films for food packaging applications. J. Agric. Food Chem. 66, 395-413 (2018).

11. Chen, Q. J., Zhou, L. L., Zou, J. Q. \& Gao, X. The preparation and characterization of nanocomposite film reinforced by modified cellulose nanocrystals. Int. J Biol. Macromol. 132, 1155-1162 (2019).

12. Chen, Z., Zhang, H., Song, Z. \& Qian, X. Preparation and application of maleic anhydride-acylated chitosan for wet strength improvement of paper. BioResources 8, 3901-3911 (2013).

13. Nicu, R., Bobu, E. \& Desbrieres, J. Chitosan as cationic polyelectrolyte in wet-end papermaking systems. Russe 45, 105-111 (2011).

14. Aloui, H., Khwaldia, K., Slama, M. B. \& Hamdi, M. Effect of glycerol and coating weight on functional properties of biopolymercoated paper. Carbohydr. Polym 86, 1063-1072 (2011)

15. Wu, M., Sukyai, P., Lv, D., Zhang, F., Wang, P. \& Liu, C. et al. Water and humidity-induced shape memory cellulose nanopaper with quick response, excellent wet strength and folding resistance. Chem. Eng. J. 392, 123673-123683 (2020).

16. Bobu, E., Nicu, R., M. Lupei, F. C. \& Desbrieres, J. Synthesis and characterization of N-alkyl chitosan for papermaking applications. Cellulose Chem. Technol 45(9-10), 619-625 (2011).

17. Stawski, D., Sahariah, P., Hjálmarsdóttir, M., Wojciechowska, D., Puchalski, M. \& Másson, M. N,N,N-trimethyl chitosan as an efficient antibacterial agent for polypropylene and polylactide nonwovens. J. Tex. I 108, 1041-1049 (2017).

18. de Britto, D. \& Assis, O. B. G. A novel method for obtaining a quaternary salt of chitosan. Carbohydr. Polym 69, 305-310 (2007).

19. Domard, A., Rinaudo, R. \& Terrassin, C. New method for the quaternization of chitosan. Int. J. Biol. Macromol. 8, 105-107 (1986)

20. Wu, M., Long, Z., Xiao, H. \& Dong, C. Preparation of N, N, N-trimethyl chitosan via a novel approach using dimethyl carbonate. Carbohydr. Polym 169, 83-91 (2017).

21. Das, S., Sharma, P. \& Vatsa, R. K. Tracing photoionisation behaviour of methyl iodide in gas phase: From isolated molecule to molecular aggregate. J Photoch. Photobio. C: Photochemistry Reviews 33, 27-53 (2017).

22. Esan, A. O., Adeyemi, A. D. \& Ganesan, S. A review on the recent application of dimethyl carbonate in sustainable biodiesel production. JClean. Prod. 257, 120561 (2020).

23. Benediktsdottir, B. E., Baldursson, O. \& Masson, M. Challenges in evaluation of chitosan and trimethylated chitosan (TMC) as mucosal permeation enhancers: From synthesis to in vitro application. J Control Release: official journal of the Controlled Release Society 173, 18-31 (2014).

24. Wu, M., Long, Z., Xiao, H. \& Dong, C. Recent research progress on preparation and application of N, N, N-trimethyl chitosan. Carbohydr. Res. 434, 27-32 (2016).

25. Santos, J. C., Moreno, P. M., Mansur, A. A., Leiro, V., Mansur, H. S. \& Pego, A. P. Functionalized chitosan derivatives as nonviral vectors: physicochemical properties of acylated N,N,N-trimethyl chitosan/oligonucleotide nanopolyplexes. Soft matter 11, 8113-8125 (2015).

26. Goy, R. C., Morais, S. T. B. \& Assis, O. B. G. Evaluation of the antimicrobial activity of chitosan and its quaternized derivative on E. coli and S. aureus growth. Rev. Bras. Farmacogn. 26, 122-127 (2016).

27. Ristić, T., Zabret, A., Zemlijič, L. F. \& Peršin, Z. Chitosan nanoparticles as a potential drug delivery system attached to viscose cellulose fibers. Cellulose 24, 739-753 (2016)

28. Chattopadhyay, D. P. \& Inamdar, M. S. Studies on the synthesis and application of N,N,N-trimethyl chitosan chloride (TMCHT) on cotton fabric. J Nat. Fibers 12, 341-356 (2015).

29. Zhang, X., Xiao, N., Wang, H., Liu, C. \& Pan, X. Preparation and characterization of regenerated cellulose film from a solution in lithium bromide molten salt hydrate. Polymers (Basel) 10, 614-627 (2018).

30. Benediktsdóttir, B. E., Gaware, V. S., Rúnarsson, Ö. V., Jónsdóttir, S., Jensen, K. J. \& Másson, M. Synthesis of N,N,N-trimethyl chitosan homopolymer and highly substituted $\mathrm{N}$-alkyl-N,N-dimethyl chitosan derivatives with the aid of di-tert-butyldimethylsilyl chitosan. Carbohydr. Polym 86, 1451-1460 (2011).

31. Lv, D., Du, H., Che, X., Wu, M., Zhang, Y. \& Liu, C. et al. Tailored and integrated production of functional cellulose nanocrystals and cellulose nanofibrils via sustainable formic acid hydrolysis: Kinetic study and characterization. ACS Sustain. Chem. Eng. 7, 9449-9463 (2019).

32. Xu, T., Xin, M., Li, M., Huang, H., Zhou, S. \& Liu, J. Synthesis, characterization, and antibacterial activity of N,O-quaternary ammonium chitosan. Carbohydr. Res. 346, 2445-2450 (2011).

33. Kulkarni, A. D., Patel, H. M., Surana, S. J., Vanjari, Y. H., Belgamwar, V. S. \& Pardeshi, C. V. Biomaterials based on N,N,N-trimethyl chitosan fibers in wound dressing applications. Int. J. Biol. Macromol. 89, 471-476 (2016).

34. Sieval, A. B., Thanoual, M., Kotze, A. F., Verhoef, J. C., Brussee, J. \& Junginger, H. E. Preparation and NMR characterization of highly substituted IV-trimethyl chitosan chloride. Carbohydr. Polym. 36, 157-165 (1998).

35. Asasutjarit, R., Theerachayanan, T., Kewsuwan, P., Veeranodha, S., Fuongfuchat, A. \& Ritthidej, G. C. Development and evaluation of diclofenac sodium loaded-N-trimethyl chitosan nanoparticles for ophthalmic use. AAPS PharmSciTech 16, 1013-1024 (2015).

36. Ramalingam, P. \& Ko, Y. T. Enhanced oral delivery of curcumin from N-trimethyl chitosan surface-modified solid lipid nanoparticles: pharmacokinetic and brain distribution evaluations. Pharm. Res. 32, 389-402 (2015).

37. de Britto, D. \& de Assis, O. B. Synthesis and mechanical properties of quaternary salts of chitosan-based films for food application. Int. J. Biol. Macromol. 41, 198-203 (2007). 
38. Kulkarni, A. D., Patel, H. M., Surana, S. J., Vanjari, Y. H., Belgamwar, V. S. \& Pardeshi, C. V. N,N,N-trimethyl chitosan: An advanced polymer with myriad of opportunities in nanomedicine. Carbohydr. Polym. 157, 875-902 (2017).

39. Kamel, S., El-Sakhawy, M. \& Nada, A. M. A. Mechanical properties of the paper sheets treated with different polymers. Thermochim. Acta 421, 81-85 (2004).

40. Nada, A. M. A., El-Sakhawy, M., Kamel, S., Eid, M. A. M. \& Adel, A. M. Mechanical and electrical properties of paper sheets treated with chitosan and its derivatives. Carbohydr. Polym 63, 113-121 (2006).

41. He, Z., Chowdhury, A., Tong, L., Reynolds, M. \& Ni, Y. Cellulose paper-based strapping products for green/sustainable packaging needs. Paper and Biomaterials 4, 54-68 (2019).

42. Liu, J., Chinga-Carrasco, G., Cheng, F., Xu, W., Willför, S., Syverud, K. et al. Hemicellulose-reinforced nanocellulose hydrogels for wound healing application. Cellulose 23, 3129-3143 (2016).

43. Yang, L., Lu, S., Li, J., Zhang, F. \& Cha, R. Nanocrystalline cellulose-dispersed AKD emulsion for enhancing the mechanical and multiple barrier properties of surface-sized paper. Carbohydr. Polym 136, 1035-1040 (2016).

44. Lee, H. L. \& Shin, J. Surface sizing with cationic starch: Its effect on paper quality and papermaking process. Tappi J 1, 34-40 (2002).

45. Tang, Y., Hu, X., Zhang, X., Guo, D., Zhang, J. \& Kong, F. Chitosan/titanium dioxide nanocomposite coatings: Rheological behavior and surface application to cellulosic paper. Carbohydr. Polym. 151, 752-759 (2016).

46. Liu, K., Chen, L., Huang, L., Ni, Y. \& Sun, B. Enhancing antibacterium and strength of cellulosic paper by coating triclosan-loaded nanofibrillated cellulose (NFC). Carbohydr. Polym 117, 996-1001 (2015).

47. Sothornvit, R. Effect of hydroxypropyl methylcellulose and lipid on mechanical properties and water vapor permeability of coated paper. Food Res. Int. 42, 307-311 (2009).

48. Mirmehdi, S., Hein, P. R. G., de Luca Sarantópoulos, C. I. G., Dias, M. V. \& Tonoli, G. H. D. Cellulose nanofibrils/nanoclay hybrid composite as a paper coating: Effects of spray time, nanoclay content and corona discharge on barrier and mechanical properties of the coated papers. Food Packaging Shelf 15, 87-94 (2018).

49. Liu, K., Xu, Y., Lin, X., Chen, L., Huang, L., Cao, S. et al. Synergistic effects of guanidine-grafted CMC on enhancing antimicrobial activity and dry strength of paper. Carbohydr. Polym. 110, 382-387 (2014).

50. Toivonen, M. S., Kurki-Suonio, S., Schacher, F. H., Hietala, S., Rojas, O. J. \& Ikkala, O. Water-resistant, transparent hybrid nanopaper by physical cross-linking with chitosan. Biomacromolecules 16, 1062-1071 (2015).

51. Lindström, T., Fellers, C., Ankerfors, M. \& Nordmark, G. G. On the nature of joint strength of paper - Effect of dry strength agents Revisiting the Page equation. Nord. Pulp Pap. Res. J31, 459-468 (2018).

52. Sharma, D. K. Effect of paper coating materials on printability. Int. J Sci., Engineering \& Computer Technology 6, 48-49 (2016).

53. Chen, Q., Dong, X., Zhou, L., Zheng, X., Wang, J. \& Wang, P. Nanostarch surface coating of lightweight coated paper. BioResources 13, 729-739 (2018).

54. El-Sherif, H. M., Nasser, A. M., Hussin, A. I., El-Wahab, H. A., Ghazy, M. B. M. \& Elsayed, A. E. Tailoring of mechanical properties and printability of coated recycled papers. Polym. Bull. 76, 2965-2990 (2019).

55. Zhang, Z., Zhang, Q., Zhang, M., Huang, R. \& Han, Y. Effect of pigment sizing on printability and coating structure of decorative base paper. Nord. Pulp Pap. Res. J33, 105-112 (2018).

56. Long, Z., Wu, M., Peng, H., Dai, L., Zhang, D. \& Wang, J. Preparation and oil-resistant mechanism of chitosan/cationic starch oilproof paper. BioResources 10, 7907-7920 (2015).

57. Velychko, O. Zolotukhina \& Rozum, T. The improvement of dampening solution for offset printing. Eastern-European Journal of Enterprise Technologies 4, 37 (2016).

58. Rossitza, S. Offset printing without isopropyl alcohol in damping solution. Energy Procedia 74, 690-698 (2015)

59. Kapoor, S. G., Wu, S. M. \& Pandit, S. M. New method for evaluating the printing smoothness of coated papers. Tappi J 61, 71-74 (1978).

60. Verheul, R. J., Amidi, M., van der Wal, S., van Riet, E., Jiskoot, W. \& Hennink, W. E. Synthesis, characterization and in vitro biological properties of Omethyl free N,N,N-trimethylated chitosan. Biomaterials 29, 3642-3649 (2008).

61. Park, J. H., Cho, Y. W., Chung, H., Kwon, I. C. \& Jeong, S. Y. Synthesis and characterization of sugar-bearing chitosan derivatives: Aqueous solubility and biodegradability. Biomacromolecules 4, 1087-1091 (2003).

62. Reis, A. B., Yoshida, C. M. P., Reis, A. P. C. \& Franco, T. T. Application of chitosan emulsion as a coating on Kraft paper. Polym. Int. 60, 963-969 (2011).

63. Vartiainen, J., Motion, R., Kulonen, H., Rättö, M., Skyttä, E. \& Ahvenainen, R. Chitosan-coated paper: Effects of nisin and different acids on the antimicrobial activity. J Appl. Poly. Sci. 94, 986-993 (2004).

\section{Acknowledgements}

This work was financially supported by the National Natural Science Foundation of China (No. 31270633), Shandong Provincial Natural Science Foundation, China (No. ZR2018BC044), Shandong Provincial Natural Science Foundation for Distinguished Young Scholar (China) (No. ZR2019JQ10), and the Foundation (Grant No. KF201802) of Key Laboratory of Pulp and Paper Science and Technology of Ministry of Education/Shandong Province of China.

\section{Author contributions}

M.W. conducted the experiments and wrote the manuscript. R.X. and C.L. analysed the results. Z.L. and B.L. supervised the work, and B.L. revised the manuscript.

\section{Competing interests}

The authors declare no competing interests.

\section{Additional information}

Correspondence and requests for materials should be addressed to B.L. or Z.L.

Reprints and permissions information is available at www.nature.com/reprints.

Publisher's note Springer Nature remains neutral with regard to jurisdictional claims in published maps and institutional affiliations. 
(c) (i) Open Access This article is licensed under a Creative Commons Attribution 4.0 International License, which permits use, sharing, adaptation, distribution and reproduction in any medium or format, as long as you give appropriate credit to the original author(s) and the source, provide a link to the Creative Commons license, and indicate if changes were made. The images or other third party material in this article are included in the article's Creative Commons license, unless indicated otherwise in a credit line to the material. If material is not included in the article's Creative Commons license and your intended use is not permitted by statutory regulation or exceeds the permitted use, you will need to obtain permission directly from the copyright holder. To view a copy of this license, visit http://creativecommons.org/licenses/by/4.0/.

(C) The Author(s) 2020 